\title{
An inventory model with dependent returns and disposal cost
}

\author{
Mohammad Ebrahim Nikoofal* and Seyed Mohammad Moattar Husseini
}

Department of Industrial Engineering,, AmirKabir University of Technology, Tehran, Iran

\section{A R T I C L E I N F O}

Article history:

Received 1 Feb 2010

Received in revised form

15 March 2010

Accepted 1 April 2010

Available online 7 April 2010

Keywords:

Reusable items

Inventory management

Reverse logistics

Dependent returns

Disposal rate

\begin{abstract}
A B S T R A C T
Environmental legislation and customer expectations force manufactures to take back their products after use. In this paper a recovery system for a single item is studied, in which the random returns depend on the demand stream. The model is different from other works in this field by considering the effect of disposal cost at the end of the planning horizon, on the total inventory cost. The proposed model of this paper explains how to change the reorder point to maintain the optimal policy. The paper is also supported with some numerical example to demonstrate the implementation of the optimal reordering policy.
\end{abstract}

\section{Introduction}

Reverse Logistics deals with activities associated with handling and management of equipment, products, components, materials or even entire technical systems which are supposed to be recovered. Global warming has been one of the most important issues and the recovery of used products and materials is believed to reduce the green gas effects. Each year, auto makers sell over sixty million cars and there is a growing concern to find suitable ways on recycling car parts remanufacturing. Electronic devices are other mass produced items where one may be more interested in reusing electronic scrap recycling. Inderfurth (1997) is believed to be the first to present a simple optimal replenishment and disposal policies for a product recovery system with lead times. Fleischman and Kuik (1998) studied an optimal inventory control with stochastic item returns. Kiesmuller and van der laan (2001) introduced an inventory model with dependent product demands and returns. Fleischman et al. (2002) introduced a basic model to control inventories with stochastic item returns. There is also a good overview on quantitative models for recovery production planning and inventory control is given in Fleischmann et al. (1997). Different papers have studied inventory policies under consideration of random returns, but most of these assume that the return process is independent of the demand process (See for example Fleischmann and Kuik (2003)). In all previous works, there is no dependency between demand and returns but there are situations where it is better to consider the probabilities of dependency between demands and returns. For example, we can think about the situations in the case of rented or leased products, or if delivered items are returned to the original manufacturer only. Cohen and Pierskalla (1980) developed an inventory model for a single, reusable product where random returns depend on the demand stream and no

* Corresponding author. Tel./fax: +1 5146492129

E-mail addresses: mohammad.nikoofal@mail.mcgill.ca (M.E. Nikoofal). 
backorders and leadtime for purchasing are considered. Also, Yuan and Cheung (1998) considered a model with dependent returns with the consideration of backorders and no purchasing lead time. De Brito and Van der laan (2009) proposed an inventory model with the uncertain time and quantity of returns. They investigated the impact of imperfect information with respect to the return process on inventory management performance. Parlikad and McFarlane (2010) proposed a model to show the value of information in product recovery decisions. Guide Jr. and Wassenhove (2009) introduced a new model for managing remanufactured products. Kiesmuller and Van der laan (2001) relaxed this assumption and allowed a positive purchasing lead time. Lieckens and Vandaele (2007) designed the reverse logistics network with stochastic lead times. The long-term behaviour of the inventory was already investigated by Van der Laan and Salomon (1998) and, in this paper we consider a finite planning horizon. The reason for this assumption is that, nowadays the life-cycle of products is getting shorter and shorter, because of fast changing trends and new developments. In this paper, we investigate an inventory model for a single, reusable product in which the random returns depend on the demand stream. We consider backorders and positive lead time for purchasing; especially we note the effect of disposal cost at the end of the planning horizon. Van der laan, Dekker and Salomon $(1996,1997)$ investigated the different inventory control systems with disposal. There are also situations that quality of recovered items is not suitable for remanufacturing process. Therefore, the rate of disposal in such situations would be increased. For example we can see this condition in car part factories. We also consider the remanufactured item as a new one. Corbacioglu and Van der laan (2007) studied the inventory model when the quality of remanufactured items was different with that of new ones. The paper is organized as follows. In section 2 we describe our model and its assumptions. In section 3 we determine the objective function and this followed by a numerical study to show importance of model in section 4. Finally, we summarize our results and give an outlook for further research.

\section{Model description}

The proposed model of this paper considers a single stage product recovery system.

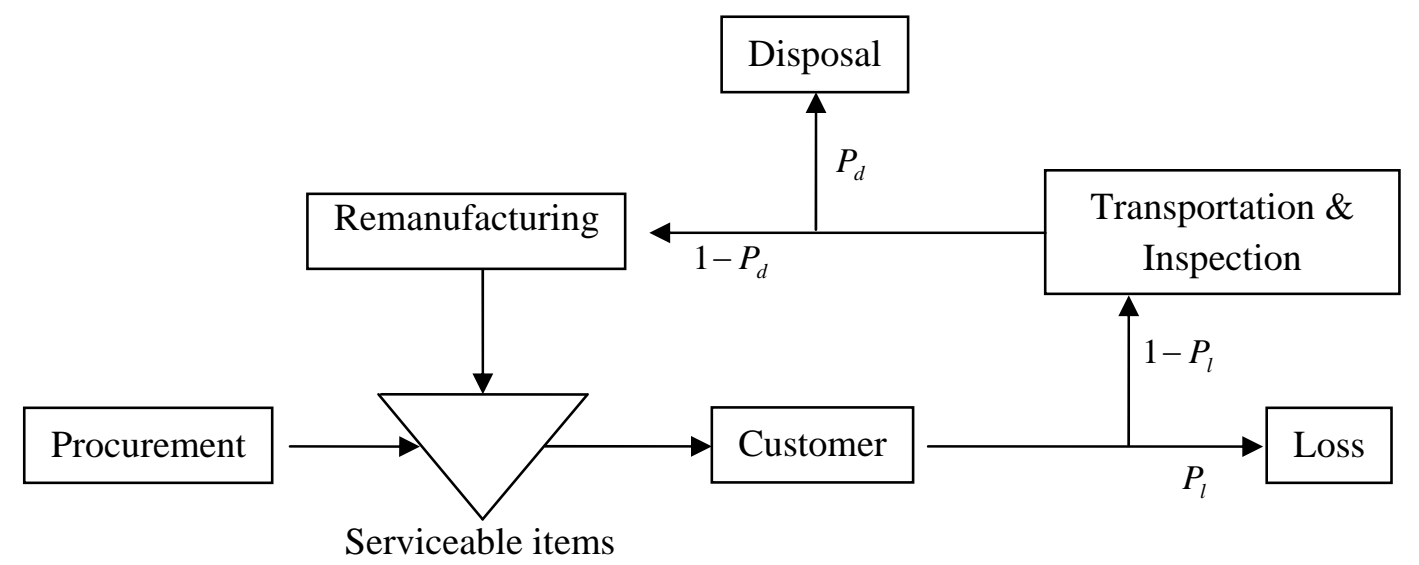

Fig 1: The reuse network

Fig. 1 demonstrates the details of the product recycling system where demand has a poison distribution over a finite planning horizon of length $\mathrm{T}(t \in[0, T])$. Practically, there are some limitations on the number of periods where inventory are reviewed. Also, for the sake of simplicity, we consider a fixed period of review to be equal to one with $t=1,2, \ldots, T$ where $t$ denotes the number of periods. Let $D_{t}$ be the demand in period $t$ with Poisson distribution and parameter $\lambda_{t}$. Therefore, we have,

$$
P\left(D_{t}=i\right)=e^{-\lambda_{t}} \cdot \frac{\left(\lambda_{t}\right)^{i}}{i !}, \quad i \in N_{0}, t=1,2, \ldots, T-1, T
$$


We assume that all demands which cannot be fulfilled immediately are backordered and the rest of outstanding demands at the end of the planning horizon are lost. The parameters in Fig. 1 are described as follow:

$P_{l}$ : Probability that customer has not returned the used product to the manufacturer.

$P_{d}$ : Probability that returned item because of its poor quality has to be disposed.

$1-P_{d}$ : Probability that returned item is remanufactured.

After remanufacturing, the item is as good as new. Therefore, there is a common serviceable inventory for both new and remanufactured items, since we do not need to distinguish between them. We assume that the lead times are constant. Let $L_{1}$ be the time where item is in use by the customer, $L_{2}$ be the transportation and the inspection time, $L_{3}$ and $L$ be the remanufacturing time and the purchasing lead time, respectively. For simplifying and computational reasons we assume that the following holds,

$L=L_{1}+L_{2}+L_{3}$

Last order with S

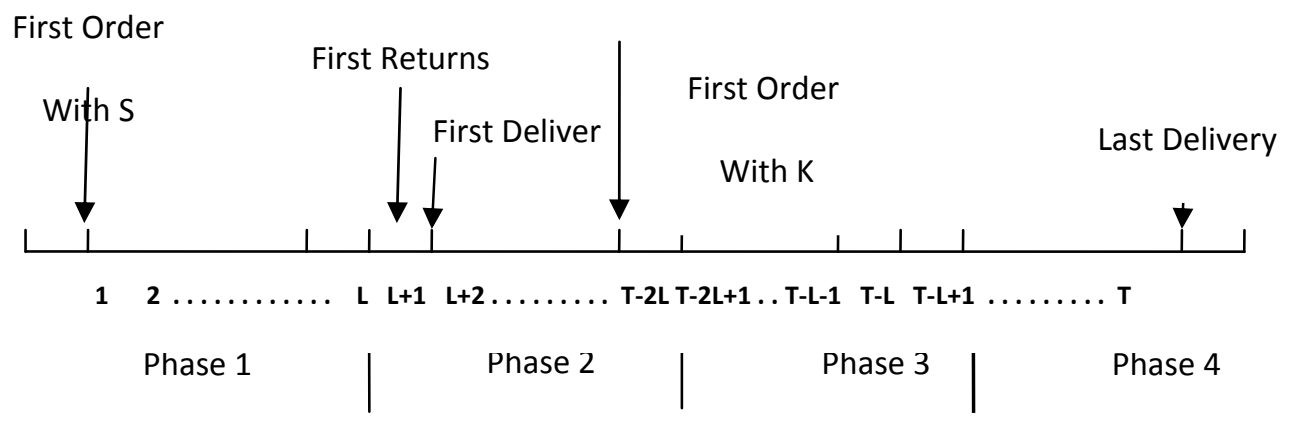

Fig 2: The time phase

Since the returns in period $t$ depends on demands for $L$ previous periods, the returns in period $t$ can be defined as the number of remanufactured items after they are used and entered to serviceable items in period $t$ based on a periodic order-up-to strategy. At the beginning of the planning horizon, the inventory is assumed to be filled up with $A$ items and an order is placed up to a limit of $S$ if the inventory position is smaller than $S$. As we can observe, two parameters of $S$ and $A$ determine our strategy. To distinguish four different time phases we assume $T \geq 3 L$. The details of our assumptions are depicted in Fig. 2.

Phase $1(1 \leq t \leq L)$ : During the periods in this phase there are no remanufactured or new items which are delivered to the serviceable items and the on hand stock decreases over this time period and the reorder point for new orders is defined by $S$.

Phase $2(L+1 \leq t \leq T-2 L)$ : In the periods of this phase both remanufactured and new items enter to the serviceable items. The stock on hand can be decreased or increased during each period in this phase. In this phase, like the phase 1 , the reorder point for new orders is defined by $S$.

Phase $3(T-2 L+1 \leq t \leq T-L)$ : Because at the end of the planning horizon (last $L$ periods), all the return items have to be disposed and on the other hand, the returns depend on the demands in $L$ periods before, so in this phase, the reorder point must be changed to decrease the cost of disposal at the end of the planning horizon and the reorder point is defined by $K$.

Phase $4(T-L+1 \leq t \leq T)$ : In this phase, no procurement orders take place. In other words, all returned items are disposed and no used items can enter to the serviceable inventory. In this model the set of all possible order-up-to policies is given by: 
In order to compare deferent parameter sets $(A, S, K) \in \xi$, we use the average total relevant cost as an objective function. So we define the average total cost by:

$C_{R}(A, S, K)=C_{0}(A)+\sum_{t=1}^{T} C_{t}(A, S, K)+C_{E}(A, S, K), \quad \forall(A, S, K) \in \xi$

They are defined as:

$C_{0}$ : The starting cost for filling up the inventory before the first period.

$C_{t}$ : The sum of the average relevant cost in period $t$.

$C_{E}$ : The average cost at the end of the planning horizon for the disposal of the remaining items.

The optimal policy $\left(A_{\text {opt }}, S_{\text {opt }}, K_{\text {opt }}\right) \in \xi$ is defined as the parameter set that minimizes expression (4). The starting expenditures solely depend on the number of items in the inventory at the beginning of the planning horizon and on some fixed ordering $\operatorname{cost} C_{A}$ as follows,

$C_{0}(A)=C_{A}+C_{P} \cdot A$

In expression (5), $C_{A}$ is the fixed ordering cost to order $A$ items and $C_{p}$ is the procurement cost for each item. The average relevant cost in one period is composed of the average costs for procurement, backorders and stock keeping and they are proportion to the number of items and the holding and the backorder costs which are charged at the end of each period. Therefore we have,

$C_{t}(A, S, K)=C_{p} \cdot E\left[O_{t}\right]+C_{B} \cdot E\left[X_{t}^{-}\right]+C_{H} \cdot E\left[X_{t}^{+}\right]$

Where the random variables are defined as follows,

$O_{t}$ : Number of procured items at the beginning of period $t$,

$X_{t}^{-}$: Number of backorders at the end of period $t$,

$X_{t}^{+}$: Stock on hand at the end of period $t$.

The cost parameters are defined as:

$C_{H}:$ Holding cost per item per period,

$C_{B}$ : Backorder cost per item per period.

For the description of the inventory process it is necessary to introduce some additional random variables:

$X_{t}:$ Net stock at the end of period $\mathrm{t} ;\left(X_{t}=X_{t}^{+}-X_{t}^{-}\right)$

$I_{t}$ : Inventory position at the beginning of period t before a procurement order,

$R_{t}$ : Number of remanufactured items which are entered to the serviceable items in period $t$.

Additionally, we define $P_{r}$ as the probability that an item is recovered, which means that it is returned to the manufacturer and then it can be remanufactured: 
$P_{r}=\left(1-P_{l}\right) \cdot\left(1-P_{d}\right)$

\section{Determination of objective function}

The objective function of the proposed model is partly formulated based on what Kiesmuller and Van der laan (2006) originally suggested. However, there are some changes on the structure of the cost items to make it compliant to our proposed method in each phase. The first step of our method is to introduce the inventory position $I_{t}$ which is explained in the next section.

\subsection{The inventory position $I_{t}$}

In the model described in Section 2, a strong relation is given between the demands in period $t$ and the returns in period $t+L$. If all the demands are fulfilled in period $t$, the distribution function of the random variable $R_{t+L}$ is only determined by the distribution function of the random variable $D_{t}$ and the recovery probability $P_{r}$. Let $\hat{R}_{t}$ be a random variable, defined as the number of returns in period $t+L$, under the condition that all the demands in period $t$ are fulfilled. Then we can use $\hat{R}_{t}$ as an approximation for $R_{t+L}$ in situations with a high service level. Practically, there is also a strong relationship between the demands in period $t$ and the returns in period $t+L$. If the demands in period $t$ are known, we have some information about the returns at $L$ later periods and the information could be used for the procurement decision in period $t+L$ and this assumption holds when we define the inventory position $I_{t}$, at the beginning of period $t$ before ordering, as the stock on hand minus backorders plus the outstanding orders plus the outstanding returns. Since we normally face a high service level, we use the following as an approximation for the inventory position for $1 \leq t \leq T-1$,

$$
I_{t+1}=X_{t}+\sum_{i=0}^{L-1} O_{t-i}+\sum_{i=0}^{L-1} \hat{R}_{t-i}
$$

or

$$
I_{t+1}=I_{t}+O_{t}-D_{t}+\hat{R}_{t}
$$

This means that the inventory position in period $t+1$ is given by the inventory position of the previous period, the procurements and the number of demands in period $t$, and the number of fulfilled demands that will return to the inventory, periods later. Therefore, $I_{t}$ describes a non-homogeneous Markov-chain, because the inventory position in period $t+1$ depends only on the period before. If we consider the dependency of the demands and the returns, $D_{t}-\hat{R}_{t}$ is always non-negative, since it is not possible to have more returns in period $t+L$. It follows from (9) that the inventory position never exceeds $S$, so that we have $I_{t} \in\{i \mid i \leq S, 1 \leq t \leq T-2 L\} \cup\{i \mid i \leq K, T-2 L+1 \leq t \leq T\}$. As a contrast, if the dependency of demands and returns is not considered, then $D_{t}-\hat{R}_{t}$ can also be negative, so that $I_{t} \in Z$. For a description of the system, we need the state probabilities $v_{j}(t)$, which are the probabilities that the inventory position in period $t$ is equal to $j$ :

$v_{j}(t)=P\left(I_{t}=j\right), \quad 1 \leq t \leq T, j \in Z$

At the beginning of period 1, both the inventory position and the stock on hand have the value $A$, which leads to:

$v_{A}(1)=1$ and $\quad v_{j}(1)=0, \quad \forall j \neq A$

All the other state probabilities are given by the recursive formula,

$v_{j}(t+1)=\sum_{i=-\infty}^{\infty} P_{i, j}(t) \cdot v_{i}(t)$ 
where $P_{i, j}(t)$ denotes the transition probability to go from state $i$ in period to state $j$ in period $t+1$,

$P_{i, j}(t)=P\left(I_{t+1}=j \mid I_{t}=i\right), 1 \leq t \leq T-1, i, j \in Z$

For the transition probabilities see Appendix A.

\subsection{The objective function}

To define the objective function we have to determine the average shortage and holding costs in period $t$ which are given for all $t=1,2,3, \ldots, T-1, T$. For average shortage cost we have,

$$
C_{H} \cdot E\left[X_{t}^{+}\right]=C_{H} \cdot \sum_{i=1}^{\infty} i \cdot P\left(X_{t}=i\right)
$$

For average holding cost we have,

$$
C_{B} \cdot E\left[X_{t}^{-}\right]=C_{B} \cdot \sum_{i=1}^{\infty} i \cdot P\left(X_{t}=-i\right)
$$

The probability distribution of the net stock $P\left(X_{t}=i\right)$ is given in Appendix A.4. Now, we have to consider the average procurement cost in the objective function. We have different procurement sizes in different periods.

$$
E\left[O_{t}\right]=\left\{\begin{array}{lc}
0, & t=1, \\
\sum_{i=-\infty}^{S-1}(S-i) \cdot v_{i}(t), & 2 \leq t \leq T-2 L, \\
\sum_{i=-\infty}^{K-1}(K-i) \cdot v_{i}(t), & T-2 L+1 \leq t \leq T-L, \\
0, & T-L+1 \leq t \leq T .
\end{array}\right.
$$

With (14), (15) and (16) the average costs in period $t, C_{t}(A, S, K)$ can be computed for $t=1,2, \ldots, T$. At the end of the planning horizon all the items in the reuse network and the items which are remained in the serviceable items must be disposed. Therefore we have,

$$
C_{E}(A, S, K)=C_{D} \cdot\left(E\left[X_{T}^{+}\right]+\left(1-P_{l}\right) \cdot \sum_{i=0}^{L_{1}+L_{2}-1} \lambda_{T-i}\right)+C_{T} \cdot\left(1-P_{l}\right) \cdot \sum_{i=1}^{L_{1}-1} \lambda_{T-i}
$$

Now with (4) we can calculate the objective function $C_{R}(A, S, K)$ for parameter sets $(A, S, K) \in \xi$. The optimal policy can be found using the optimal solution of the following problem,

Min $C_{R}(A, S, K)$

$(A, S, K) \in \xi$

Since the structure of objective function is nonlinear, a closed form solution cannot be found easily and use numerical techniques, instead.

\section{Numerical study}

In this section, we use a numerical example to demonstrate the implementation of the proposed method of this paper. We consider three cases: For the first case, we study the model when we have only one reorder point for all periods $t=1,2, \ldots, T$. In the second case, we have two reorder points of $1 \leq t \leq T-2 L$ and $T-2 L+1 \leq t \leq T$. Also, demand and return sales are assumed to be constant. The other parameters are as follows, 


$$
\begin{aligned}
& \lambda=3, \quad T=24, \quad P_{l}=0, \quad L_{1}=1, \quad L_{2}=1, \quad L_{3}=1, \quad C_{B}=50, \\
& C_{D}=20, \quad C_{P}=50, \quad C_{H}=1, \quad C_{A}=0 .
\end{aligned}
$$

In Table 1 the optimal policies and the average minimal relevant costs are given for different values of $P_{d}$ and $P_{r}$. Also the Relative difference between two systems is given, which shows the difference between the average minimal costs of each system.

\section{Table 1}

\begin{tabular}{|c|c|c|c|c|c|c|c|c|c|}
\hline \multirow{2}{*}{$P_{r}$} & \multirow{2}{*}{$P_{d}$} & \multicolumn{3}{|c|}{ Ordering system with $S$} & \multicolumn{4}{|c|}{ Ordering system with $S, K$} & \multirow{2}{*}{$\begin{array}{l}\text { Relative } \\
\text { difference } \\
(\%)\end{array}$} \\
\hline & & $A_{o p t}$ & $S_{\text {opt }}$ & $C_{R}\left(A_{o p t}, S_{o p t}\right)$ & $A_{o p t}$ & $S_{\text {opt }}$ & $K_{o p t}$ & $C_{R}\left(A_{o p t}, S_{o p t}, K_{o p t}\right)$ & \\
\hline 0 & 0 & 16 & 16 & 1489 & 16 & 16 & 13 & 1355 & 9.9 \\
\hline 0.25 & 0.25 & 17 & 17 & 2294 & 17 & 17 & 13 & 2117 & 8.3 \\
\hline 0.5 & 0.5 & 18 & 18 & 3102 & 18 & 18 & 14 & 2890 & 7.3 \\
\hline 0.75 & 0.75 & 19 & 19 & 3916 & 19 & 19 & 14 & 3655 & 6.8 \\
\hline
\end{tabular}

Optimal policy and the minimal average relevant costs to compare two different systems

\section{Conclusion and future research}

In this paper, we have developed an inventory system with periodic ordering policy which the return stream is depended on the demand stream. We consider the finite planning horizon and the backorders are allowed. We have studied the effect of recovery probability on determining the optimal policy and the average total cost. We show that with considering the cost of disposal at the end of the planning horizon, the reorder point should be changed to achieve the optimal policy. There are some limitations on the assumptions of the proposed model. For instance, we have considered only one inventory for the serviceable items. It is worth to be discussed, that a remanufactured item does not necessarily have the same quality as the new one. In this case, the recovery probability for an item is not constant but decreasing. Therefore, one can define two inventories for serviceable items, one inventory for new items and another for the remanufactured items. Moreover, it would be interesting to extent the model with the stochastic lead time structure.

\section{Acknowledgment}

The authors would like to sincerely thank the anonymous referees whose comments improved the earlier version of this paper.

\section{Appendix A}

\section{A.1 The distribution of the returns $\hat{R}_{t}$ and of $D_{t}-\hat{R}$}

If $P_{r}$ denote the probability that an item is recovered $\left(P_{r}=\left(1-P_{l}\right) \cdot\left(1-P_{d}\right)\right)$, then we get the following formula for the distribution of the returns, $\hat{R}_{t}$ for all $t=L+1, L+2, \ldots, T-1$ :

$$
\begin{aligned}
P\left(\hat{R}_{t}=i\right) & =\sum_{j=i}^{\infty} P\left(\hat{R}_{t}=i \mid D_{t-L}=j\right) P\left(D_{t-L}=j\right)=\sum_{j=i}^{\infty}\left(\begin{array}{l}
j \\
i
\end{array}\right) P_{r}^{i}\left(1-P_{r}\right)^{j-i} \cdot e^{-\lambda_{t-L}} \cdot \frac{\lambda_{t-L}^{j}}{j !} \\
& =e^{-\lambda_{t-L}} \frac{P_{r}^{i}}{i !} \sum_{j=i}^{\infty} \frac{j !}{(j-i) !}\left(1-P_{r}\right)^{j-i} \frac{\lambda_{t-L}^{j}}{j !}=e^{-\lambda_{t-L} \frac{\left(P_{r} \cdot \lambda_{t-L}\right)^{i}}{i !} \sum_{j=i}^{\infty} \frac{\left(\lambda_{t-L}\left(1-P_{r}\right)\right)^{j-i}}{(j-i) !}} \\
& =e^{-\lambda_{t-L}} \frac{\left(P_{r} \lambda_{t-L}\right)^{i}}{i !} e^{\left(1-P_{r}\right) \lambda_{t-L}}=e^{-P_{r} \lambda_{t-L}} \frac{\left(P_{r} \lambda_{t-L}\right)^{i}}{i !}
\end{aligned}
$$


Therefore, the number of returns in period $t$ is distributed with Poison distribution and parameter $P_{r} \lambda_{t-L}$.

$\hat{R}_{t} \sim \operatorname{Po}\left(P_{r} . \lambda_{t-L}\right), \quad t=L+1, L+2, \ldots, T-1$

The distribution is independent of $t$, if $\lambda_{t}=\lambda$. For $t=1,2, \ldots, L-1$ and for $t=T$, there are no returns, i.e. $\hat{R}_{t}=0$. The distribution of the number of items which are not returned $D_{t}-\hat{R}_{t}$ can be determined similarly. Therefore we have,

$D_{t}-\hat{R}_{t} \sim \operatorname{Po}\left(\lambda_{t}\left(1-P_{r}\right)\right)$

\section{A.2 The distribution of $\sum_{i=0}^{L} D_{t-i}-\hat{R}_{t}$}

In order to determine the distribution of $\sum_{i=0}^{L} D_{t-i}-\hat{R}_{t}$ we have to consider that the random variables $\hat{R}_{t}$ and $D_{t-L}$ are dependent, while the other random variables not. We can write:

$$
\sum_{i=0}^{L} D_{t-i}-\hat{R}_{t}=\underbrace{D_{t-L}-\hat{R}_{t}}_{\text {dependent }}+\underbrace{\sum_{i=0}^{L-1} D_{t-i}}_{\text {independent }}
$$

where $D_{t-L}-\hat{R}_{t}$ is Poisson distributed with parameter $\lambda_{t-L}\left(1-P_{r}\right)$,

$D_{t-L}-\hat{R}_{t} \sim \operatorname{Po}\left(\lambda_{t}\left(1-P_{r}\right)\right)$

and $\sum_{i=0}^{L-1} D_{t-i}$ is Poisson distributed with parameter $\sum_{i=0}^{L-1} \lambda_{t-i}$,

$\sum_{i=0}^{L-1} D_{t-i} \sim P o\left(\sum_{i=0}^{L-1} \lambda_{t-i}\right)$

$D_{t-L}-\hat{R}_{t}$ and $\sum_{i=0}^{L-1} D_{t-i}$ itself are independent. Therefore, the sum of these terms is again Poisson distributed, which means:

$\sum_{i=0}^{L} D_{t-i}-\hat{R}_{t} \sim P o\left(\lambda_{t-L}\left(1-P_{r}\right)+\sum_{i=0}^{L-1} \lambda_{t-i}\right)$

\section{A.3 The transition probabilities}

Because of the non-homogeneous Markov-chain the transition probabilities are dependent on time.

$t=1$ : The initial distribution (11) and the fact that $D_{t}-\hat{R}_{t}$ is always non-negative lead to

$P_{i, j}=\left\{\begin{array}{lr}P\left(D_{1}-\hat{R}_{1}=A-j\right), & j \leq A, n=A \\ 0, & \text { elsewhere }\end{array}\right.$

and the state probability

$v_{j}(2)=P\left(D_{1}-\hat{R}_{1}=A-j\right)$

$2 \leq t \leq T-2 L$ : In these periods from all the demands a portion will be returned to the inventory $L$ period later, which means $\hat{R}_{t} \geq 0$. Additionally, the inventory position after an order is always $S$, so that $I_{t}+O_{t}=S$.Therefore, we have 
$P_{i, j}=P\left(D_{t}-\hat{R}_{t}=S-j\right)$

These transition probabilities are independent from $i$ and the state probabilities are given by

$v_{j}(t+1)=P\left(D_{t}-\hat{R}_{t}=S-j\right)$

$T-2 L+1 \leq t \leq T-L$ : The transition probabilities of these periods are like above but instead of $S$ we use $K$. Therefore we have,

$P_{i, j}=P\left(D_{t}-\hat{R}_{t}=K-j\right)$

and regard to A.11 we have

$v_{j}(t+1)=P\left(D_{t}-\hat{R}_{t}=K-j\right)$

$t=T-L$ : For $t=T-L$, we still have procurement, but none of the delivered items to the customer will be returned to the serviceable inventory $\left(\hat{R}_{t-L}=0\right)$. Therefore, we get

$P_{i, j}(T-L)=P\left(D_{T-L}=K-j\right)$

and

$v_{j}(T-L+1)=P\left(D_{T-L}=K-j\right)$

$T-L+1 \leq t \leq T-1$ : In these periods we have again $\hat{R}_{t}=0$ but there are no orders $\left(O_{t}=0\right)$, which leads to

$P_{i, j}(t)=P\left(D_{t}=K-j\right)$

\section{A.4 The distribution of the net stock}

Different formulas are needed for different periods.

$1 \leq t \leq L$ : Because of no returns in Phase 1 , the net stock only depends on the demand and is monotonously decreasing. There are also no deliveries of new items. For $i \in \mathrm{Z}$ it holds that

$P\left(X_{t}=i\right)=P\left(\sum_{k=1}^{t} D_{k}=A-i\right) \quad t=1,2, \ldots, L$

These probabilities can easily be computed, since the sum of independent Poisson distributed random variables is again Poisson distributed:

$\sum_{k=1}^{t} D_{k} \sim P o\left(\sum_{k=1}^{t} \lambda_{k}\right)$

$L+1 \leq t \leq T-1$ : In Phases 2, 3 and 4 the net stock at the end of period $t$ is dependent on the inventory position in period $t-L$ :

$$
X_{t}=I_{t-L}+O_{t-L}+\hat{R}_{t}-\sum_{k=0}^{L} D_{t-k}, \quad L+1 \leq t \leq T
$$

For the probabilities we have to distinguish between $t=L+1$ and $t \neq L+1$.

$t=L+1$ : In this case there are no procurements $\left(O_{1}=0\right)$ and the inventory position in period 1 is given by $I_{1}=A$. Therefore, we get 
$P\left(X_{L+1}=i\right)=P\left(\sum_{k=0}^{L} D_{L+1-k}-\hat{R}_{L+1}=A-i\right)$

$t \neq L+1$ : In this case the probabilities for the net stock can be computed as follows:

$P\left(X_{t}=i\right)=P\left(\sum_{k=0}^{L} D_{t-k}-\hat{R}_{t}=S-i\right) \quad 1 \leq t \leq T-2 L, t \neq L+1$

and

$P\left(X_{t}=i\right)=P\left(\sum_{k=0}^{L} D_{t-k}-\hat{R}_{t}=K-i\right), \quad T-2 L+1 \leq t \leq T-1$

$t=T$ : For $t=T$ the random variable $\hat{R}_{t}$ in (A.19) is equal to 0 and we get

$P\left(X_{T}=i\right)=P\left(\sum_{k=0}^{L} D_{T-k}=K-i\right)$

\section{References}

de Brito, M. P. \& Van der laan, E. A. (2009). Inventory control with product returns: The impact of imperfect information. European Journal of Operational Research, 194, 85-101.

Corbacioglu, U. \& Van der laan, E. (2007). Setting the holding cost rates in a two-product system with remanufacturing, International of Production Economics, 109, 185-194.

Cohen, M. A., \& Pierskalla, W. P. (1980). A dynamic inventory system with recycling, Naval Research Logistics Quarterly, 27, 289-296.

Fleischmann, M., Bloemhof-Ruwaard, J. M., Dekker, R., van der Laan, E. A., van Nuenen, J. A. E. E. \& van Wassenhove, L. N. (1997). Quantitative models for reverse logistics: A review, European Journal of Operational Research 103, 1-17.

Fleischman, M. \& Kuik, R. (1998). On optimal inventory control with stochastic item returns, Management Report, Erasmus University Rotterdam, 21.

Fleischmann, M., \& Kuik, R. (2003). An optimal inventory control with independent stochastic item returns, European journal of Operational Research, 151, 25-37.

Fleischman, M., Kuik, R., \& Dekker, R. (2002). Controlling inventories with stochastic item returns: A basic model, European Journal of Operational Research, 138, 63-75.

Guide Jr., V. D. R. \& Wassenhove, L. V. (2009). Managing product returns for remanufacturing, Production and Operation Management, 10, 142-155.

Inderfurth, K., (1997). Simple optimal replenishment and disposal policies for a product recovery system with lead times, OR Spektrum, 19, 111-122.

Kiesmuller, G. P., \& van der laan, E. A. (2001). An inventory model with dependent product demands and returns, International Journal of Production Economics, 72, 73-87.

Lieckens K., \& Vandaele, N. (2007). Reverse logistics network design with stochastic lead times, Computers \& Operations Research, 34, 395-416.

Parlikad, A. K. \& McFarlane, D. (2010). Value of information in product recovery decisions: A Bayesian approach. International Journal of Sustainable Engineering. DOI: 10.1080/19397030903499810.

Van der laan, E., Dekker, R. \& Salomon, M. (1996), Product remanufacturing and disposal: A numerical comparison of alternative control strategies, International Journal of Production Economics, 45, 489-498.

Van der laan, E. \& Salomon, M., (1997). Production planning and inventory control with remanufacturing and disposal, European Journal of Operational Research, 102, 264-278.

Yuan, X. \& Cheung, K. (1998). Modelling returns of merchandise in an inventory system, OR Spektrum, 20, $147-154$ 